\title{
Effect of spectral broadening and electron-hole scattering on carrier relaxation in GaAs quantum dots
}

\author{
Igor Vurgaftman and Jasprit Singh \\ Solid State Electronics Laboratory, Department of Electrical Engineering and Computer Science, \\ The University of Michigan, Ann Arbor, Michigan 48109-2122
}

(Received 13 August 1993; accepted for publication 2 November 1993)

\begin{abstract}
Luminescence efficiency in quantum dots has been a matter of some controversy recently. Theoretically, poor efficiency has been predicted owing to the phonon bottleneck in carrier relaxation, while slightly enhanced luminescence has been reported in several experiments. The approach of this letter differs from previous theoretical work in that the scattering rates are computed self-consistently accounting for the spectral broadening of the electronic spectra due to a finite energy level lifetime. Scattering of electrons and holes confined in the dot is found to be responsible for breaking the phonon bottleneck in electron relaxation reducing the relaxation time from several ns to several hundred ps. Results of a Monte Carlo simulation also including confined and interface polar optical phonon and acoustic phonon scattering for a range of quantum dot dimensions and temperatures are presented. These results may provide an explanation of the absence of a significant reduction in quantum dot luminescence compared with that from quantum wells.
\end{abstract}

The optoelectronic properties of quasi-one-dimensional electronic systems have been investigated recently for potential applications in ultralow threshold semiconductor lasers ${ }^{1}$ and with regard to the predicted enhancement in optical nonlinearities. ${ }^{2}$ The realization is growing, however, that although the discrete eigenvalue spectrum in quantum dots results in a greatly increased oscillator strength for optical transitions, coupling of electrons to polar optical phonons (POP) may be drastically reduced in small dots, and as a result, relaxation of electrons to the ground state may be hindered. Phonon scattering rates in quantum dots have been studied by Bockelmann and Bastard. ${ }^{3}$ They pointed out that unless the electron states are separated by the energy of the optical phonon, the mechanism for POP scattering in quantum dots is different from that in bulk semiconductors. Electron relaxation may occur as a result of phonon renormalization, broadening of the electron and phonon spectrum, emission of short-wavelength acoustic phonons, or higher order phonon scattering processes. Considering electron relaxation by emission of acoustic phonons, Benisty et al. ${ }^{4}$ have predicted poor radiative efficiency in quantum dots with lateral dimensions less than $500 \AA$ in consequence of the electron relaxation times being comparable to nonradiative recombination times (a few ns). Although a number of experiments have been reported which appear to substantiate this conclusion, ${ }^{5-7}$ several workers have observed luminescence in very small quantum dots, in which the effect of the phonon bottleneck should become noticeable. ${ }^{8,9}$ Recently enhanced luminescence has been observed in 35-nm InGaAs/ GaAs quantum dots defined by a combination of electron beam epitaxy, dry ctching, and molecular beam epitaxial regrowth, ${ }^{10}$ although fabrication-induced changes in the potential profile in those structures may be substantial. In experimentally realizable quantum dots, nonradiative recombination may be enhanced by controllable defects as well as by the relaxation bottleneck; therefore, the issue of electron relaxation in quantum dots cannot be considered well understood at present.
In this letter, we present a theoretical analysis of electron relaxation in $\mathrm{GaAs} / \mathrm{Al}_{0.3} \mathrm{Ga}_{0.7} \mathrm{As}$ quantum dots based on a Monte Carlo simulation of the equilibration process with the inclusion of self-consistently calculated electron-phonon and electron-hole scattering processes. An idealized flat-band potential profile is assumed, i.e., no attempt is made to describe the band-bending properties of the $\mathrm{GaAs} / \mathrm{AlGaAs}$ system resulting from a particular fabrication process. The electronic spectrum is found by solving the Schrödinger equation in the effective mass approximation, and the hole states are derived by diagonalizing the four-band Kohn-Luttinger Hamiltonian, in which the standard prescription for calculating quantum confined states $\left[k_{x} \rightarrow-i(\partial / \partial x)\right.$, etc. $]$ is used. In order to estimate the electron-hole scattering rate, for the sake of computational simplicity, the off-diagonal coupling terms in the Hamiltonian are set to zero. The eigenenergies and eigenfunctions are found by discretizing the volume of the dot on a uniform spatial mesh terminated far away from the dot interfaces, approximating derivatives as finite differences, and solving the resulting matrix equation iteratively.

The macroscopic electrostatic model has proved useful in approximating the spatial structure and dispersion relations for phonon models in quantum wells and wires. ${ }^{11} \mathrm{We}$ assume that the contined phonon modes are given by linear combinations of products of sines and cosines, whose wave vector is fixed at multiples of $\pi / L_{i}$, where $i=x, y, z$, by imposing the boundary condition that the phonon mode vanish near the interface with a material with a different resonant vibration frequency. Since the nonepitaxial dimensions greater than $20 \mathrm{~nm}$ are examined in this letter, we assume quantum well interface phonon modes varying as hyperbolic sines and cosines with the dispersion relation derivable from the boundary condition. ${ }^{11}$ The interface phonon energies lie in the Reststrahlen region between the transverse $\left(\hbar \omega_{\mathrm{TO}} \approx 33\right.$ $\mathrm{meV}$ in GaAs) and longitudinal ( $\left.\hbar \omega_{\mathrm{LO}} \approx 36 \mathrm{meV}\right)$ optical phonon energies.

The confined POP scattering rate is calculated using the 
Fröhlich form of the interaction Hamiltonian from the Fermi Golden Rule:

$$
\begin{aligned}
W_{i f}^{\mathrm{POP}}= & \frac{2 \pi}{\hbar} \sum_{l, m, n} \frac{e^{2} \hbar \omega_{\mathrm{LO}}}{2 L_{x} L_{y} L_{z}}\left(\frac{1}{\epsilon_{\infty}}-\frac{1}{\epsilon_{0}}\right) \\
& \times\left[\left(\frac{\pi l}{L_{x}}\right)^{2}+\left(\frac{\pi m}{L_{y}}\right)^{2}+\left(\frac{\pi n}{L_{z}}\right)^{2}\right]^{-1} I_{i f}^{2} \\
& \times\left(n\left(\omega_{\mathrm{LO}}\right)+\frac{1}{2} \pm \frac{1}{2}\right)\left|M_{i f}^{\mathrm{POP}}(l, m, n)\right|^{2} \\
& \times \delta\left(E_{f}-E_{i} \pm \hbar \omega_{\mathrm{LO}}\right),
\end{aligned}
$$

where $I_{i f}$ is the overlap integral for the cell periodic parts of the electron wave function, presumed unity in this work, and the rest of the symbols have their usual meaning. The upper sign corresponds to phonon emission, the lower sign to absorption. Taking an arbitrary vertex of the box as origin, the overlap integral for the envelope parts of the wave function can be written down simply as

$M_{i f}^{\mathrm{POP}}(l, m, n)=\int d^{3} r \varphi_{f}^{*}(\mathbf{r}) \sin \left(\frac{l \pi x}{L_{x}}\right) \sin \left(\frac{m \pi y}{L_{y}}\right) \sin \left(\frac{n \pi}{L_{z}}\right) \varphi_{i}(\mathbf{r})$.

The delta function in Eq. (1) is replaced by a Gaussian line shape function:

$$
\delta\left(E_{f}-E_{i} \pm \hbar \omega_{\mathrm{LO}}\right) \rightarrow \frac{1}{\sqrt{1.44 \pi \sigma}} \exp \left(-\frac{\left(E_{f}-E_{i} \pm \hbar \omega_{\mathrm{LO}}\right)^{2}}{1.44 \sigma^{2}}\right)
$$

An iterative self-consistent calculation of the scattering rates and the linewidth $\sigma=\hbar / \tau$, where $\tau$ is taken to be the reciprocal of the scattering rate, is necessary to determine $W_{i f}^{\text {POP }}$ from Eqs. (1)-(3). The scattering rates have also been calculated for interface phonon modes and have been found to be lower by two orders of magnitude than the rates for confined bulklike phonons. Consequently, even for phonon energies in the range between 33 and $36 \mathrm{meV}$, confined phonon scattering dominates, with the energy difference made up by the broadening of the electronic spectrum. By assumption, the lattice is maintained at a constant temperature.

In quantum wires, acoustic phonon (AP) scattering is one of the mechanisms responsible for electron relaxation for quantum wircs of cross sections less than $100 \AA \times 100 \AA .^{12} \mathrm{In}$ quantum dots, the AP scattering rate can be calculated using the Fermi Golden Rule and the deformation potential theory:

$$
\begin{aligned}
W_{i f}^{\mathrm{AP}}= & \frac{\pi}{\rho L_{x} L_{y} L_{z}} \sum_{\mathbf{q}} \frac{D_{A}^{2} q^{2}}{\omega_{\mathbf{q}}}\left(n\left(\omega_{\mathbf{q}}\right)+\frac{1}{2} \pm \frac{1}{2}\right) \\
& \times\left|\int d^{3} r \varphi_{f}^{*}(\mathbf{r}) e^{i \mathbf{q} \cdot \mathbf{r}} \varphi_{i}(\mathbf{r})\right|^{2} \delta\left(E_{f}-E_{i} \pm \hbar \omega_{\mathbf{q}}\right),
\end{aligned}
$$

where $\rho$ is the mass density of GaAs, $D_{A}$ is the acoustic deformation potential, and $q$ is the phonon wave vector with a continuous spectrum. We include acoustic phonon scattering in our Monte Carlo simulation, although in small quantum dots, the energy levels are separated by energies available only to short-wavelength AP. The matrix element for short phonon wavelength averages out to nearly zero, and the $\mathrm{AP}$ rates are by several orders of magnitude smaller than

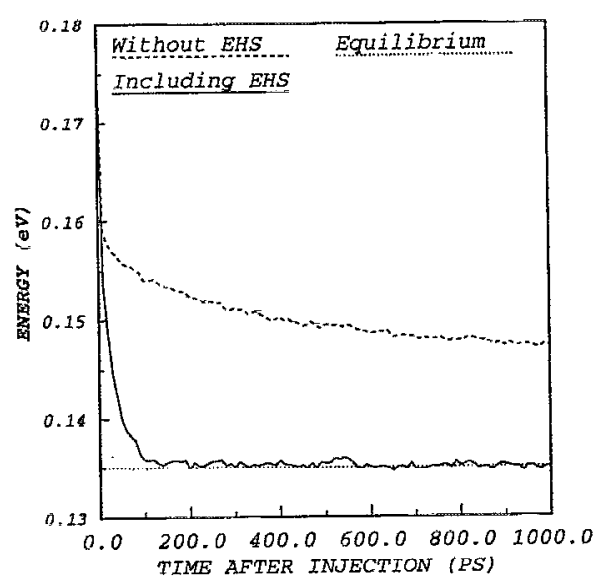

FIG. 1. The mean electron energy as a function of time after injection for the $50 \AA \times 250 \AA \times 250 \AA \mathrm{GaAs} / \mathrm{Al}_{0.3} \mathrm{Ga}_{0.7}$ As quantum dot at $300 \mathrm{~K}$. The horizontal line represents the equilibrium mean energy. The averaging is over 5000 carriers.

POP modes. Due to the weaker coupling of acoustic phonons to electrons, the broadening linewidth is also much smaller than that for POP.

The Pauli exclusion principle has to be considered in the occupation of discrete quantum dot levels. However, because of the small size of dots under study, it is permissible to assume that only one electron is injected in any one quantum dot. With this assumption, the electron-electron interaction and screening can be ignored. Previous treatments of electron relaxation mechanisms in quantum dots have not included electron-hole (EH) scattering. Since the hole confinement energy is much smaller than that for the electron, the hole distribution can be assumed to be in thermal equilibrium with the lattice. Therefore, the electron may lose energy by interacting with a thermalized hole in the valence band. The matrix element can be computed in the Born approximation:

$$
\begin{aligned}
M_{i f}^{\mathrm{EH}}= & \int d^{3} r_{e} \int d^{3} r_{h} \sum_{\nu=1} \varphi_{f}^{* e}\left(\mathbf{r}_{\mathrm{e}}\right) \varphi_{f}^{* h, \nu}\left(\mathbf{r}_{h}\right) \frac{e^{2}}{4 \pi \epsilon} \\
& \times \frac{1}{\left|\mathbf{r}_{e}-\mathbf{r}_{h}\right|} \varphi_{i}^{e}\left(\mathbf{r}_{e}\right) \varphi_{i}^{h, \nu}\left(\mathbf{r}_{h}\right),
\end{aligned}
$$

where the sum runs over the $|3 / 2, \pm 3 / 2\rangle$ and $|3 / 2, \pm 1 / 2\rangle$ angular momentum states. The scattering rate is calculated by applying the Fermi Golden Rule with the broadening found in an iterative fashion as described above.

Given the relevant scattering rates, electron relaxation can be modeled by a Monte Carlo simulation. Electrons are injected at the top of the potential barrier in a thermal distribution, and their evolution is followed in energy and time. An averaging over a large number of electrons (and equivalently, dots) is necessary in order to produce statistically reliable results. In Fig. 1 the evolution of the mean energy of the electron distribution in the $50 \AA \times 250 \AA \times 250 \AA$ $\mathrm{GaAs} / \mathrm{Al}_{0.3} \mathrm{Ga}_{0.7} \mathrm{As}$ quantum dot at $300 \mathrm{~K}$ is shown. In absence of EH scattering, the main energy loss mechanism is POP emission with a lifetime-broadened energy spectrum. Nevertheless, POP scattering is unable to provide coupling between states whose separation is different from $36 \mathrm{meV}$ by 


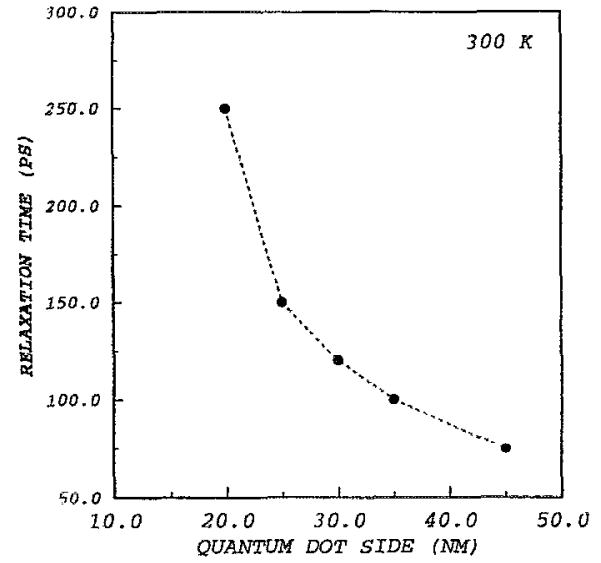

FIG. 2. The dependence of the relaxation time on the side of the square cross section of a GaAs/Al $/ 0.3 \mathrm{Ga}_{0.7} \mathrm{As}$ quantum dot with a width of $50 \AA$ in the direction of epitaxial growth at $300 \mathrm{~K}$. Data points are indicated by bullets.

more than several meV. As a result, the relaxation time reaches several ns, thus becoming comparable with the nonradiative recombination time in most samples. However, if EH scattering is taken into account, the bottleneck is broken, and the clectron relaxation time decreascs to a little over 100 ps. At lower temperatures, phonon and $\mathrm{EH}$ scattering are more efficient since fewer phonons are available for absorption, and the hole distribution is sharper. Therefore, the effect of EH scattering is similar yet more pronounced, with a relaxation time of less than $30 \mathrm{ps}$ at $70 \mathrm{~K}$.

A brief comment on the accuracy of the assumption of the equilibrium hole distribution is in order. Since the hole confinement energies are much smaller than the electron confinement energies, and the hole density of states is much higher than the electron density of states, the hole spectrum is broadened as to be nearly continuous. In these circumstances, hole relaxation is likely to resemble relaxation of electrons in quantum wires, for which the relaxation time does not exceed $100 \mathrm{ps.}^{12}$ Since we have estimated the electron relaxation times in quantum dots to be of the order of several hundred ps, the effect of hot holes on electron relaxation is secondary.

The dependence of the relaxation time on the cross section by a plane perpendicular to the direction of epitaxial growth in shown in Fig. 2. In larger quantum boxes, a greater number of states are available for occupation in the conduction and valence bands; therefore, relaxation is bound to proceed at a higher rate. As the lateral dimensions of the dot increase further, the equilibration time further decreases until the time for the capture of electrons from extended states into quantum-confined states begins to dominate the relaxation time in the limit of the quantum well structure. The dimensions of the $\mathrm{GaAs} / \mathrm{Al}_{0.3} \mathrm{Ga}_{0.7} \mathrm{As}$ dot cannot be reduced below $20 \mathrm{~nm}$ if any bound states are to exist below the potential barrier. For stronger confining potentials, quantum dots with 5-nm size quantization in all three dimensions are theoretically possible; however, the relaxation processes based on the lifetime broadening are too weak to insure efficient electron and hole equilibration. Unless a qualitatively different energy loss mechanism is discovered, relaxation times in such structures may be extremely long.

In brief summary, we have presented the results of a Monte Carlo simulation of electron relaxation in $20-45 \mathrm{~nm}$ quantum dots with the inclusion of the broadening of the energy spectrum and coupling to confined and interface phonon modes and of the consequences of electron-hole scattering. The latter is the mechanism principally responsible for breaking the bottleneck which occurs as a result of a sharp LO phonon spectrum and inefficiency of acoustic phonon scattering. The electron relaxation time in a $50 \AA \times 250$ $\AA \times 250 \AA$ dot has been estimated to be several hundred ps at room temperature. The equilibration time is reduced by lowering temperature and expanding the size of the dot. However, it remains much larger than the relaxation time in quantum wires (several tens of ps) at $300 \mathrm{~K}$. Therefore, the modulation properties of quantum dot lasers, for which very low threshold currents have been predicted, can be impaired.

This work was supported by the Army Research Office under Contract No. DAAL 03-92-G-0109.

${ }^{1}$ Y. Arakawa and H. Sakaki, Appl. Phys. Lett. 40, 939. (1982).

${ }^{2}$ S. Schmitt-Rink, D. Miller, and D. Chemla, Phys. Rev. B 35, 8113 (1987).

${ }^{3}$ U. Bockelmann and G. Bastard, Phys. Rev. B 42, 8947 (1990).

${ }^{4}$ H. Benisty, C. Sotomayor-Torres, and C. Weisbuch, Phys. Rev. B 44, 10945 (1991).

${ }^{5}$ P. Wang, C. Sotomayor-Torres, H. Benisty, C. Weisbuch, and S. Beaumont, Appl. Phys. Lett. 61, 946 (1992).

${ }^{6}$ C. Sotomayor-Torres, W. Leitch, D. Lootens, P. Wang, G. Williams, S. Thomas, H. Wallace, P. Van Daele, A. Cullis, C. Stanley, P. Demeester, and S. Beaumont, in Nanostructures and Mesoscopic Systems (Academic, San Diego, CA, 1992), p. 455.

${ }^{7}$ A. Forchel, B. E. Maile, H. Leier, G. Mayer, and R. Germann, in Science and Engineering of One- and Zero-Dimensional Semiconductors (Plenum, New York, 1990), p. 277.

${ }^{8}$ Y. Nagamune, M. Nishioka, S. Tsukamoto, and Y. Arakawa, in Proceedings of the 19th International Symposium on GaAs and Related Compounds (Karuizawa, Japan, 1992) (IOP, Bristol, UK, 1993), p. 335.

${ }^{9}$ H. Temkin, G. Dolan, M. Panish, and S. Chu, Appl. Phys. Lett. 50, 413 (1987).

${ }^{10}$ I. Davis, K. Ko, W. Li, H. Sun, Y. Lam, T. Brock, S. Pang, and P. Bhattacharya, Appl. Phys. Lett. 62, 2766 (1993).

${ }^{11} \mathrm{~J}$. Likari and R. Evrard, Phys. Rev. B 15, 2254 (1977).

${ }^{12}$ I. Vurgaftman and J. Singh, Appl. Phys. Lett. 62, 2251 (1993). 